Revue internationale P.M.E.

\title{
Les stratégies de coopération des galeries d'art contemporain
}

l'apport de l'économie des proximités

Shedding light on cooperative strategies of contemporary art

galleries

\author{
A proximity approach
}

\section{Estrategias de cooperación de galerías de arte contemporáneo contribución de la economía de proximidad}

\author{
Nathalie Moureau, Dominique Sagot-Duvauroux et Muriel de Vrièse
}

Volume 27, numéro 3-4, 2014

URI : https://id.erudit.org/iderudit/1028043ar

DOI : https://doi.org/10.7202/1028043ar

Aller au sommaire du numéro

Éditeur(s)

Editions EMS - In Quarto SARL

ISSN

0776-5436 (imprimé)

1918-9699 (numérique)

Découvrir la revue

Citer cet article

Moureau, N., Sagot-Duvauroux, D. \& de Vrièse, M. (2014). Les stratégies de coopération des galeries d'art contemporain : l'apport de l'économie des proximités. Revue internationale P.M.E., 27(3-4), 125-143.

https://doi.org/10.7202/1028043ar

\section{Résumé de l'article}

Cet article a pour objectif d'étudier dans quelles conditions la proximité spatiale entre galeries d'art est susceptible de déboucher sur des coopérations. L'hypothèse que nous cherchons à tester est que les proximités spatiale et cognitive doivent s'accompagner d'une proximité de valeurs pour que naissent des stratégies collaboratives. L'économie des proximités constitue le corpus théorique principal sur lequel nous nous appuyons. Une double enquête, quantitative, à partir des données de la Maison des artistes, et qualitative, à partir d'entretiens semi-directifs réalisés auprès des acteurs de l'art actuel dans cinq métropoles françaises, nous permet de constater que les galeries d'art sont extrêmement concentrées sur le territoire français sans que cette proximité spatiale ne se traduise nécessairement par des stratégies coopératives, alors même qu'elles partagent le même métier et savoir-faire (proximité cognitive). Nous mettons en évidence l'existence de différents réseaux de l'art actuel au sein d'un même espace géographique. Nous montrons que ces réseaux se différencient principalement par le fait qu'ils ne partagent pas les mêmes critères d'appréciation de la qualité des oeuvres (conventions de qualité artistique). 


\title{
Les stratégies de coopération des galeries d'art contemporain : l'apport de l'économie des proximités
}

\author{
Nathalie MOUREAU
}

Nathalie Moureau est maître de conférences en sciences économiques et chercheure au LAMETA, Université de Montpellier. Elle est spécialisée en économie de la culture et a publié plusieurs articles et ouvrages sur le sujet. Elle a notamment publié Le marché de l'art contemporain aux éditions La Découverte (collection Repères).

LAMETA, Université Montpellier

UFR d'Économie

Avenue Raymond Dugrand - Site de Richter C.S. 79606

34960 MONTPELLIER CEDEX 2

nathalie.moureau@univ-montp3.fr

\section{Dominique SAGOT-DUVAUROUX}

Dominique Sagot-Duvauroux est professeure en sciences économiques et chercheure au GRANEM, Université d'Angers. Elle est spécialisée en économie de la culture et a publié plusieurs articles et ouvrages sur le sujet. Elle a notamment publié Le marché de l'art contemporain aux éditions La Découverte (collection Repères).

\author{
GRANEM Université d'Angers \\ 13, allée François Mitterrand \\ 49036 ANGERS CEDEX 01 \\ dominique.sagot-duvauroux@univ-angers.fr
}

\section{Muriel DE VRIÈSE}

Muriel De Vrièse est maître de conférences en sciences économiques et chercheure au CERENE, Université du Havre. Elle est spécialisée en économie de la culture et a publié plusieurs articles et ouvrages sur le sujet.

Université du Havre 25, rue Philippe Lebon

76600 LE HAVRE muriel.de_vriese@yahoo.fr

\section{RÉSUMÉ}

Cet article a pour objectif d'étudier dans quelles conditions la proximité spatiale entre galeries d'art est susceptible de déboucher sur des coopérations. L'hypothèse que nous cherchons à tester est que les proximités spatiale et cognitive doivent s'accompagner d'une proximité de valeurs pour que naissent des 
stratégies collaboratives. L'économie des proximités constitue le corpus théorique principal sur lequel nous nous appuyons. Une double enquête, quantitative, à partir des données de la Maison des artistes, et qualitative, à partir d'entretiens semi-directifs réalisés auprès des acteurs de l'art actuel dans cinq métropoles françaises, nous permet de constater que les galeries d'art sont extrêmement concentrées sur le territoire français sans que cette proximité spatiale ne se traduise nécessairement par des stratégies coopératives, alors même qu'elles partagent le même métier et savoir-faire (proximité cognitive). Nous mettons en évidencel'existence de différents réseaux de l'art actuel au sein d'un même espace géographique. Nous montrons que ces réseaux se différencient principalement par le fait qu'ils ne partagent pas les mêmes critères d'appréciation de la qualité des cuvres (conventions de qualité artistique).

MOTS CLÉS

TPE, Galerie d'art, Art actuel, Territoire, Proximités, Conventions

\title{
Shedding light on cooperative strategies of contemporary art galleries: a proximity approach
}

\begin{abstract}
The aim of this article consists in studying under which conditions spatial proximity favours cooperation between galleries. Our hypothesis is that collaborative strategies appear only when spatial and cognitive proximities are completed by proximity of value. The economy of proximity is the main theoretical corpus on which we rely. Both quantitative and qualitative database were settled. Whereas quantitative data were obtained through the database of La Maison des artistes, qualitative data were obtained through semi -structured interviews we conducted with contemporary art protagonists in five French cities. We show a high concentration of art galleries on the French territory. Nevertheless, even though some galleries share the same profession and skills (cognitive proximity), the spatial proximity they share does not lead necessarily towards cooperative strategies. We highlight the existence of different contemporary art networks within a same geographical area. We show the role played by artistic conventions for the consistency of these networks, the criteria for assessing the quality of works (conventions of artistic quality) being specific to each network.
\end{abstract}

KEYWORDS

Micro enterprises, Art gallery, Contemporary art, Territorial, Proximities, Conventions

\section{Estrategias de cooperación de galerias de arte contemporáneo : contribución de la economia de proximidad}

\begin{abstract}
RESUMEN
Este artículo tiene como objetivo el estudio de las condiciones de cooperación cuando existe una proximidad espacial entre galerías de arte. Nuestra hipótesis es que las proximidades espaciales y cognitivas deben ser acompañadas por una proximidad de los valores para la aparición de estrategias de colaboración. Este trabajo se basa en una investigación dual. Se utilizó una base de datos cuantitativa, compilada a partir de informaciones recogidas en la Maison des artistes, también hemos llevado un trabajo cualitativo, elaborado a partir de entrevistas semi-estructuradas con actores de arte contemporáneo en cinco metrópolis. Se demuestra que las galerías de arte están muy centradas en el territorio francés. Sin
\end{abstract}


embargo, esta proximidad espacial no conduce necesariamente al desarrollo de cooperaciones, a pesar de que las galerías que participan compartan la misma profesión y las mismas habilidades (proximidad cognitiva). Se destaca la existencia de diferentes redes de arte contemporáneo dentro de la misma área geográfica. Mostramos que estas redes se diferencian principalmente por el hecho de que ellos no comparten los mismos criterios para evaluar la calidad de las obras (convenciones de calidad artística).

\section{Palabras clave}

Microempresas, Galeria de arte, Arte contemporáneo, Territorio, Proximidades, Convenciones

\section{INTRODUCTION}

Enchères millionnaires, ventes médiatisées, telle est l'image de l'art contemporain que donnent à voir les transactions réalisées par ces grandes sociétés que sont Sotheby's et Christie's. Pour autant, les enchères ne constituent qu'une part réduite des transactions de l'art contemporain et, sauf cas exceptionnel (telle la vente de Damien Hirst chez Sotheby's en septembre 2008) ne concernent que le second marché, à savoir celui de la revente d'œuvres (Kusin et Company, 2005 ; Moureau et Sagot-Duvauroux, 2010). Les acteurs cruciaux du marché sont les galeries d'art qui jouent un rôle central pour la découverte de nouveaux talents (Moulin, 1992 ; Moureau et Rivaud-Danset, 2010). La façon dont elles tirent parti du territoire ${ }^{1}$ pour réduire le risque lié à leur activité n’a pas été étudiée si ce n’est à travers des études portant sur des données sectorielles agrégées comme dans les travaux conduits par Greffe et Simonnet (2008). Ceux-ci montrent que le taux de survie des entreprises culturelles est très sensible à leur regroupement géographique, l'effet de concurrence entre entreprises de même activité étant contrebalancé par des effets de synergies imputables à la présence d'autres entreprises culturelles (notion de districts culturels). Les auteurs constatent toutefois un profil assez atypique des galeries d'art qui ont un taux d'incidence ${ }^{2}$ supérieur à celui de l'ensemble des autres secteurs (culturels ou non) ${ }^{3}$.

1 Le territoire est une notion complexe. Défini comme un espace physique délimité, il se présente comme un ensemble de ressources (géographiques, humaines, économiques) dont les acteurs résidant dans ce territoire vont bénéficier. Il renvoie au concept de proximité spatiale qui favorise les échanges physiques entre individus. Défini comme un espace construit par les acteurs, il se présente comme un réseau d'acteurs liés entre eux par des proximités autres que spatiales par exemple, cognitives, sociales ou culturelles. Notre approche du territoire croise ces deux définitions et s'intéresse aux intersections entre territoires physiques, socio-économiques ou culturels. À quelles conditions l'appartenance à un même espace physique favorise-t-elle l'appartenance à un espace économique de collaboration ? (Bellet, Colletis et Lung, 1993).

2 i.e. pourcentage des entreprises ayant cessé leur activité dans les cinq ans relativement au nombre total d'années d'exercice considérées.

3 Ce taux est similaire pour les entreprises culturelles prises dans leur ensemble et les entreprises des autres secteurs (soit 12,3\% pour le secteur culturel en général et $13 \%$ pour les autres secteurs), il s'établit en revanche à $15 \%$ pour les galeries d'art. La fragilité des galeries est encore plus marquée en début de période : le taux d'incidence au bout de 2 ans est de $18,25 \%$ pour les arts visuels contre $13,8 \%$ pour les autres secteurs non culturels et est de $17,66 \%$ entre 2 et 4 ans pour les arts visuels contre $14,62 \%$ pour les autres secteurs (Greffe et Simonnet, 2008). 
Étant donné le rôle central que jouent les galeries d'art pour le dynamisme du marché, analyser la façon dont elles se saisissent du territoire, comme cela a été fait pour les métiers d'art (Loup, 2003 ; Loup, Jourdain, Kosianski et Rakotovahino, 2010 ; Kosianski, 2011) ou pour le secteur touristique (Jackson et Murphy, 2002 ; Gundolf et Jaouen, 2005 ; Gundolf, 2006 ; Gundolf, Jaouen et Loup, 2006 ; Gundolf, 2008), et parviennent ou non à « travailler leur marché $»^{4}$ pourrait donner quelques éléments de réflexion quant aux actions à mettre en œuvre pour consolider le secteur.

L'objectif de cet article consiste à étudier dans quelles conditions la proximité spatiale des galeries d'art est susceptible de déboucher sur des coopérations. Après avoir dans un premier temps expliqué la dynamique qui conduit à la forte concentration des galeries sur le territoire, nous montrons que la proximité spatiale ainsi générée ne débouche pas nécessairement sur le développement de stratégies coopératives entre les galeries, tandis que celles-ci partagent le même métier et le même savoir-faire (proximité cognitive). L'hypothèse que nous cherchons à tester est que les proximités spatiale et cognitive doivent s'accompagner d'une proximité de valeurs pour que puissent naître des stratégies collaboratives. Nous commençons par effectuer dans la section 2 un rappel de la littérature afin d'asseoir théoriquement le découpage entre proximités géographiques et non géographiques qui sert de fil directeur à la suite de notre analyse. Dans la section 3, nous précisons la méthodologie qui a été suivie pour la collecte et le traitement des données (i.e. exploitation statistique des données issues de la Maison des artistes ${ }^{5}$ et enquête qualitative avec entretiens semi-directifs auprès des diffuseurs de cinq agglomérations : Le Havre, Lyon, Montpellier, Nantes et Rouen) ${ }^{6}$. La section suivante (4), présente les résultats tandis que la dernière section (5) discute tant la concentration géographique des firmes autour de certains pôles que la difficulté de certaines galeries à collaborer entre elles. Nous montrons alors comment les stratégies développées sont contingentes du type d'art pratiqué. Tandis que les galeries proposant un art traditionnel ont une occupation que l'on pourrait qualifier de plutôt passive du territoire, les galeries qui défendent un art de recherche et pour lesquelles les proximités non spatiales sont cruciales fonctionnent en réseau et contribuent à leur tour à façonner le territoire.

\section{CADRE THÉORIQUE}

Plusieurs auteurs ont montré comment le recours à la proximité facilitait l'action et la réflexion pour des organisations peu formalisées et dotées de systèmes d'information simples

4 Cette formule est empruntée à Dubuisson-Quellier (2003) lorsqu'il évoque dans ses travaux la capacité qu'ont les TPE d'agir sur leur marché.

5 La Maison des artistes est l'organisme qui est en charge en France du régime de sécurité sociale des artistes et auprès duquel toutes les structures diffusant de l'art contemporain sont tenues d'apporter leur contribution.

6 Notons que ces collectes de données sont issues d'une étude sur la valorisation et la diffusion de l'art contemporain que nous avons réalisée par ailleurs pour le département des études de la prospective et des statistiques (DEPS) du ministère de la Culture (De Vrièse, Martin, Moureau et Sagot-Duvauroux, 2011a, b). 
tels que les TPE (voir l'ouvrage coordonné par Jaouen et Torrès, 2008). L’usage de liens de proximité par les TPE pour modeler leur marché n'est toutefois pas l'apanage de toutes les structures, comme le montre Mallard (2007), qui met en exergue des structures «sans calcul », i.e. des organisations pour lesquelles le marché semble relever d'une extériorité sur laquelle elles ne cherchent pas à peser, et attribue ce comportement à une culture professionnelle où le calcul et où la rationalisation font en grande partie défaut. Sont recensées dans cette catégorie, une artiste peintre, ainsi qu'une galerie d'art (Mallard, 2007). Fillis (2002) relève également l'existence d'une certaine fatalité dans les rapports de certains acteurs au marché, en particulier chez les dirigeants d'entreprises artistiques qui adhèrent à la philosophie art for art's sake7. D’autres travaux conduits sur les métiers d'art (Loup, 2003 ; Loup et al., 2010 ; Kosianski, 2011) soulignent comment la réussite de ces « entrepreneurs de la singularité » est tributaire des liens de proximité établis entre les acteurs au sein d'un territoire où d'un métier. Comme l'ont montré ces auteurs, le rôle joué par les institutions pour favoriser l'émergence ou la consolidation des proximités géographiques, mais également les représentations et stratégies collectives, sont essentiels.

Conscientes tant de l'effet bénéfique des proximités pour le développement d'activités, que du rôle moteur que pouvaient avoir les institutions pour les impulser, de nombreuses villes ont initié dans les années 1990 le regroupement d'activités culturelles et la création de clusters culturels et créatifs pour asseoir une nouvelle orientation économique (Pilati et Tremblay, 2007 ; Scott et Leriche, 2005 ; Santagata, 2006 ; Scott, 2010). Dès 1890, Marshall avait vu dans les phénomènes d'agglomération la possibilité d'économies externes dues au développement d'un bassin de main-d'œuvre spécialisée, à l'existence d'économie de spécialisation et à l'existence d'externalités d'information et de connaissance (atmosphère industrielle). Ce n'est toutefois qu'à partir des années 1970 que la notion de performance des territoires a véritablement donné lieu à des développements théoriques. Districts, système productif local, learning region, proximités, clusters, les appellations sont multiples et traduisent tant le dynamisme de la réflexion théorique que les antagonismes potentiels qui existent entre chercheurs du fait de présupposés méthodologiques distincts (Chesnel, Molho, Morteau et Raimbeau, 2013). Trois questions ont été principalement abordées (Gordon et McCann, 2000). Quelles forces poussent les activités d'une même filière à se regrouper dans l'espace? Dans quelle mesure les relations inter-organisationnelles favorisent-elles l'efficacité du cluster et des organisations qui le composent ? Quels rôles jouent les réseaux individuels dans la dynamique collective?

À l'interface de ces questions, une communauté scientifique s'est créée dans les années 1990 autour de la thématique des proximités (Bouba-Olga et Grossetti, 2008) qui « [...] n’a cessé de produire des propositions théoriques et des ouvertures disciplinaires, intégrant sur la base de l'économie régionale et de l'économie industrielle des apports de la sociologie économique et urbaine, de la géographie économique, ou de la gestion. Il est donc dans la vocation de ce groupe de chercher régulièrement à raffiner les outils théoriques, pas nécessairement dans une logique de sophistication d'un modèle unique, mais plutôt dans un mouvement de confrontation dialectique de lignes de théorisation, ancrées dans des traditions un peu différentes (pour faire simple, plus holistes pour certains, plus interactionnistes pour d'autres), mais respectant

7 Selon l'expression employée par R. Caves (2000). 
toujours la distinction initiale et la nécessité de définir des proximités non restreintes à l'espace physique» (p. 312) .

L'économie des proximités distingue les proximités spatiales et organisées (Rallet et Torre, 2004 ; Torre et Rallet, 2005). La proximité spatiale (ou géographique) peut se définir comme " la distance kilométrique entre deux types d'entités (e.g. individus, organisations, villes) pondérée par le coût temporel et monétaire de son franchissement (Torre, 2006); elle peut être permanente (co-localisation) ou temporaire (TIC ou rencontres physiques temporaires). Cette distance dépend non seulement de paramètres objectifs mais également du jugement que portent les individus sur la distance géographique qui les sépare. Par contraste, la proximité non spatiale ou organisée n'est pas d'essence géographique mais relationnelle. Elle renvoie à la « capacité qu'offre une organisation de faire interagir ses membres. L'organisation facilite les interactions en son sein, en tout cas, les rend a priori plus faciles qu'avec les membres situés à l'extérieur de l'organisation " (Rallet et Torre, 2004, p. 37). Les regroupements sont ici effectués selon une logique de similitude ou d'appartenance. Dans le premier cas, les individus ont des connaissances communes (proximité cognitive) ou des valeurs et des références communes (proximité institutionnelle ou de valeur) qui favorisent leur capacité à interagir, dans le second, la proximité est initiée par les interactions interindividuelles survenues du fait de l'appartenance des individus à une même organisation (proximité organisationnelle) ou à un même groupe social (proximité sociale) (Boschma, 2005).

Dans cette perspective, nous allons montrer dans la suite de cet article comment interfèrent ces différents types de proximité dans le développement de coopération entre les galeries d'art actuel. La forte concentration géographique de celles-ci s'explique par les effets d'agglomération, mais cette proximité spatiale ne suffit pas à engendrer des projets coopératifs. Ceux-ci nécessitent l'existence d'une proximité organisée au sein de laquelle la proximité de valeurs s'avère déterminante.

\section{MÉTHODOLOGIE}

Cet article s'appuie sur une double enquête quantitative et qualitative. Les données quantitatives sont issues de l'exploitation des fichiers de la Maison des artistes sur trois années 2004, 2005 et 2006. Cet organisme chargé de la collecte des cotisations sociales des artistes et des diffuseurs $^{9}$ comptabilise à ce jour 2242 actifs au titre du $1 \%$ sur le chiffre d'affaires ou sur la commission sur ventes. Ces diffuseurs peuvent être des galeries, des brocantes, des antiquaires, des sociétés de ventes volontaires (SVV), des éditeurs d'art, des commerces (bars,

8 Les recherches sur les économies de proximité se sont développées en France à partir du milieu des années 1990 à l'initiative des chercheurs du groupe «Dynamiques de proximité » (voir par exemple les numéros spéciaux de la RERU, 1993 et 2008 ; et les travaux de synthèse de Rallet et Torre, 1995, 2004 ; Torre, 2006 ; Bellet, Kirat et Largeron, 1998 ; Gilly et Torre, 2000 ; Pecqueur et Zimmermann, 2004 ; Talbot et Kirat, 2005 ; Bouba-Olga et Grosseti, 2008).

9 Notons que dans ce système, des cotisations sont également prélevées sur la vente d'œuvres d'artistes décédés, cotisations qui servent ensuite à alimenter les indemnités versées aux artistes vivants. 
restaurants, magasins de meubles, etc.) et dans de rares cas, des musées ou centres d'art ${ }^{10}$. Ont été exclus de notre étude les diffuseurs qui valorisent uniquement le travail d'artistes décédés ainsi que les sociétés de ventes volontaires et les commerces (e.g. marchands de meubles). Sur cette base, un échantillon représentatif de 516 diffuseurs a été constitué en tenant compte de la localisation géographique et du chiffre d'affaires en $2006^{11}$.

Ces données ont été utilisées (statistiques descriptives) pour identifier les phénomènes de concentration géographique et les potentiels traits distinctifs des galeries selon leur localisation géographique (e.g. chiffre d'affaires, portefeuille d'artistes représentés, âge des galeries).

En parallèle, un travail plus qualitatif a été conduit sur la base d'entretiens semi-directifs auprès de l'ensemble des diffuseurs et institutions localisés dans les villes de Nantes, Le Havre, Montpellier, Lyon et Rouen (galeries, centres d'art, FRAC, musées, salons, collectivités publiques, collectionneurs); 132 entretiens ont été réalisés (voir tableau 1). Dans chaque agglomération, nous avons interrogé les principaux acteurs de l'art actuel, toutes tendances esthétiques confondues, sur les caractéristiques de leur activité ${ }^{2}$ et les relations de coopérations qu'ils entretenaient avec d'autres acteurs locaux, nationaux ou internationaux (vernissages, édition de catalogues en commun, coproductions, etc.).

TABLEAU 1. Nombre D'ENTRETIENS RÉALISÉS SUIVANT LES TERRITOIRES

\begin{tabular}{lccrc}
\hline & $\begin{array}{l}\text { Rouen/ } \\
\text { Le Havre }\end{array}$ & Montpellier & Nantes & Lyon \\
Galeries commerciales & 10 & 7 & 6 & 18 \\
Galeries associatives & 9 & 8 & 6 & 9 \\
$\begin{array}{l}\text { Collectivités territoriales (Ville, } \\
\text { Communauté de Communes, Région, }\end{array}$ & 4 & 3 & 5 & 2 \\
$\begin{array}{l}\text { Département) } \\
\text { Centre d'art conventionné }\end{array}$ & 1 & 1 & 1 & 2 \\
\hline État (DRAC) & 1 & 1 & 1 & 1 \\
\hline Institutions (FRAC, écoles d'art, musées) & 6 & 3 & 4 & 3 \\
\hline Autres (agents d'art, associations, & 5 & 5 & 8 & 4 \\
curators, collectionneurs, critiques) & & & & \\
\hline TOTAL & 36 & 28 & 31 & 37 \\
\hline
\end{tabular}

10 Notons que certains points de vente ne disposent pas d'une déclaration propre s'ils sont rattachés à une société mère ; dans ce cas, une seule déclaration est nécessaire, ce qui amoindrit en partie la population totale des diffuseurs inscrits à la MDA.

11 Moins de 1000 euros, de 1001 à 5000 euros, de 5001 à 10000 euros, de 10001 à 15 000, de 15001 à 50000 euros, plus de 50000 euros.

12 Les informations collectées avaient trait à l'identité générale de la structure (année de création ; statut juridique ; localisation ; autres implantations ; nombre de salariés, etc.), à son activité de valorisation (participation à des foires; organisation d'expositions ; édition de catalogues; nombre d'artistes locaux représentés, vivants ou morts ; modes de découverte de nouveaux artistes, etc.), à sa situation économique (nombre de visiteurs par semaine et leur origine; répartition du chiffre d'affaires par zone géographique, entre artistes vivants et décédés, selon le type d'acheteurs, etc.). 
Les réponses aux questions portant sur les collaborations ont été quantifiées ${ }^{13}$ et nous ont permis de cartographier à l'aide du logiciel Ucinet la matrice des coopérations entre les acteurs. Ce travail nous a permis d'identifier les acteurs dont le rôle était le plus central dans le réseau et ceux ayant le betweeness ${ }^{14}$ le plus élevé. La partie plus qualitative des entretiens a été utilisée pour sa part à des fins d'illustration après retranscription, lecture critique et classement.

\section{RÉSULTATS}

Nous présentons d'abord les résultats issus de l'analyse des données de la Maison des artistes qui révèlent la forte concentration géographique des galeries d'art. Dans un second temps, à partir de l'exploitation de l'enquête régionale, nous mettons en évidence l'existence de cloisonnements plus ou moins marqués entre galeries.

\subsection{Proximités géographiques : le pouvoir attractif de Paris}

En dépit de la volonté affichée des pouvoirs publics de favoriser la diffusion de l'art contemporain sur l'ensemble du territoire français dans les années 1980 à travers la création des Fonds régionaux d'art contemporain ${ }^{15}$, l'examen de la répartition des structures de diffusion marchandes montre que le marché de l'art demeure très largement l'apanage de la capitale et dans une moindre mesure, des villes à fort potentiel touristique.

\section{LOCALISATION GÉOGRAPHIQUE ET PÔLES D'ATTRACTION}

Inégalement répartis sur le territoire français, les diffuseurs d'art contemporain sont très concentrés puisque 42,6 \% d'entre eux sont localisés en Île-de-France. La région ProvenceAlpes-Côte d'Azur (14,1 \%) vient ensuite, suivie par Rhône-Alpes (6,8 \%), la Bretagne (5 \%) et l'Aquitaine (5\%). Nombreuses sont les régions où l'art actuel est très faiblement diffusé (Corse, Picardie, Franche-Comté, Champagne-Ardenne, Auvergne, Limousin, Lorraine, Centre, Antilles-Guyane-Réunion). L'analyse de la répartition du chiffre d'affaires des galeries sur le territoire semble encore plus inégale. Les structures parisiennes réalisent en effet à elles seules plus des deux tiers du chiffre d'affaires total avec une moyenne de 818460 euros par galerie contre 146239 euros en région. En moyenne, un diffuseur situé en région réalise un chiffre d'affaires 5,6 fois moins élevé qu'un de ses confrères parisiens.

13 Chaque coopération a été considérée comme un lien quelle que soit sa nature. Si plusieurs coopérations ont eu lieu entre deux partenaires, le nombre de liens n'a pas été sommé. De ce fait, l'analyse a mis en évidence l'existence de liens mais non leur force.

14 Le betweeness mesure la capacité d'un nœud de réseau à être un passage obligé.

15 Les FRAC sont des institutions non lucratives chargées de soutenir la création et de constituer au sein de chaque région un patrimoine d'art contemporain. 
En région, les grandes villes attirent un grand nombre de galeries. Par exemple, Lyon accueille 42,9 \% des galeries d'art de la Région Rhône-Alpes. Mais ce sont surtout dans les lieux touristiques que l'on recense le plus grand nombre de structures. Par exemple, pour la région PACA, les plus fortes concentrations se constatent dans les villes touristiques avec Saint-Paul-de-Vence (16,4\% des galeries régionales), Nice (15,1\%), Marseille (9,6\%), Cannes $(5,5 \%)$ et Saint-Rémy-de-Provence (5,4 \%). Le phénomène est similaire en Bretagne avec une plus forte concentration des diffuseurs sur les villes touristiques de Dinar, Dinan, Pont-Aven et Saint-Malo que sur les villes de Brest, Rennes et Quimper.

TABleAu 2. Chiffre D'AFFAIRES MOYEN DES DIFFUSEURS PAR TERRITOIRE (MOYENNE SUR 3 ANS)

\begin{tabular}{lrrrrrr}
\hline Région & Effectif & $\begin{array}{l}\text { Moyenne } \\
\text { (en euros) }\end{array}$ & $\begin{array}{l}\text { Écart-Type } \\
\text { (en euros) }\end{array}$ & $\begin{array}{l}\text { Min } \\
\text { (en euros) }\end{array}$ & $\begin{array}{l}\text { Max } \\
\text { (en euros) }\end{array}$ \\
\hline Aquitaine & 70 & 155871,2 & 291 904,4 & 430 & 1283818 \\
\hline Basse-Normandie & 46 & $\mathbf{5 0 7} \mathbf{5 6 8 , 2}$ & 1447289,0 & 88 & 88 \\
\hline Bourgogne & 42 & 126702,1 & 250054,3 & 195 & 195 \\
\hline Nord-Pas-De-Calais & 30 & 79585,3 & 67380,7 & 3900 & 3900 \\
\hline Rhône-Alpes & 98 & 123752,8 & 189325,7 & 148 & 148 \\
\hline Provence-Alpes-Côtes d'Azur & 200 & 158663,5 & 275908,3 & 89 & 89 \\
\hline Languedoc Roussillon & 44 & 79605,9 & 107238,7 & 1640 & 1640 \\
\hline Pays de la Loire & 38 & 90459,3 & 134518,9 & 708 & 708 \\
\hline Paris & 569 & $\mathbf{8 1 8} \mathbf{4 5 9 , 9}$ & 1755682,0 & 125 & 125 \\
\hline Île de France (hors Paris) & 78 & 154430,1 & 189249,5 & 871 & 871 \\
\hline Bretagne & 73 & 94702,1 & 125311,7 & 830 & 830 \\
\hline Haute-Normandie & 30 & 96698,6 & 87743,5 & 4615 & 4615 \\
\hline Midi-Pyrénées & 50 & 115144,7 & 157781,3 & 744 & 744 \\
\hline Autres régions & 112 & 114625,0 & 289652,5 & 0 & 0 \\
\hline Total & 1480 & 404680,7 & 1178273,0 & 0 & 1283818 \\
\hline
\end{tabular}

Au sein même des villes, la répartition est aussi inégale. Dans la capitale, des effets d'agglomération apparaissent autour de divers quartiers ${ }^{16}$. Les quartiers Est sont ainsi très largement délaissés $(8,9 \%)$ au profit du $6^{\mathrm{e}}$ arrondissement $(38,4 \%$ ), du quartier Beaubourg Marais (30,5\%) et du quartier Matignon (22,1\%). En région, les galeries à vocation commerciale sont majoritairement localisées dans les quartiers animés des villes, que ce soit les centres historiques (à Montpellier et à Nantes) ou le quartier des antiquaires (à Rouen et à Lyon) ; les galeries de nature associative sont en revanches localisées en périphérie ou dans des quartiers moins animés.

16 Voir sur le cas new-yorkais : Molotch et Treskon, 2009 ; Zukin, 2011. 


\section{UNE GESTION DIFFÉRENCIÉE DES RELATIONS AVEC LES ARTISTES SELON LES PÔLES}

Parallèlement à leur recherche de proximité vis-à-vis de leur clientèle, les galeries tendent à privilégier des ressources locales en représentant en priorité des artistes résidant à une faible distance géographique. Ainsi, 62,8\% des artistes dont les œuvres sont vendues par les galeries parisiennes vivent dans la région parisienne. Ce pourcentage est plus ou moins marqué selon les quartiers. La proportion d'artistes « locaux » est plus importante dans les structures des quartiers Est (77\%), puis Beaubourg-Marais $(68,7 \%)$ et enfin dans les structures de la Rive gauche $(62,4 \%)$; elle est beaucoup moins élevée dans les quartiers de Matignon $(51,6 \%)^{17}$. À l'échelle régionale, plus de la moitié des artistes résident dans la même région que leur structure de diffusion (soit 51,6 \%). Il existerait donc un véritable marché des artistes « régionaux » sur lequel les diffuseurs appuieraient une partie de leur activité.

On note également une différenciation quant à la nature des artistes representés. Tandis que dans le quartier Matignon les galeries constituent des portefeuilles mixtes d'artistes (vivants/décédés), dans les quartiers Est, les structures privilégient la vente exclusive d'œuvres d'artistes vivants (80,9\%). Cette dichotomie se retrouve également dans le nombre d'artistes représentés. Tandis que les galeries situées dans le quartier Matignon affichent à la fois les chiffres d'affaires les plus élevés ${ }^{18}$ et le nombre le moins important d'artistes représentés (2,7 en moyenne par galerie), les quartiers Est présentent en revanche un portefeuille d'artistes assez étoffé $(14,8)^{19}$.

Un différentiel de même nature apparaît entre les galeries parisiennes prises dans leur ensemble et les galeries situées en région. Ces dernières proposent un éventail d'artistes plus large que les galeries parisiennes (10,4 en moyenne contre 7 à Paris). Sans doute, les galeries qui centrent leur travail de diffusion sur l'art vivant cherchent-elles à compenser le risque différemment. Ne pouvant tirer parti de la sécurité offerte par la vente d'œuvres d'artistes décédés, dont la cote est a priori plus établie, elles joueraient sur la compensation des risques en s'appuyant sur un nombre plus important d'artistes.

17 Notons que cette concentration des galeries en Île-de-France est corrélée à une forte concentration des artistes puisqu'à peu près la moitié des plasticiens, graveurs et peintres et $36 \%$ des sculpteurs inscrits à la Maison des artistes résident en Île-de-France.

18 Il apparaît que la corrélation positive existante entre le fait de vendre les œuvres d'artistes décédés et le chiffre d'affaires est une spécialité parisienne que l'on ne retrouve ni en province ni en petite couronne. Dans le Nord-Pas-de-Calais, où les structures sont plus âgées, aucune n'atteint le seuil de $250000 €$ de chiffre d'affaires. Au contraire, dans les Pays de la Loire où l'on constate l'âge moyen le plus faible de l'échantillon (9,9 ans), 22,2\% des structures ont un chiffre d'affaires supérieur à $250000 €$. Il en va de même pour la région PACA avec un âge moyen de 11,7 ans où plus d'un quart des structures réalise un chiffre d'affaires supérieur à $250000 €$. Peut-être peut-on voir là un effet de paroi propre aux TPE jouant sur les effets de proximité qui de ce fait se satisfont d'un marché local et brident ainsi leur croissance.

19 Les galeries de la Rive gauche $(6,4)$ ou du quartier Beaubourg-Marais $(7,8)$ sont dans une position intermédiaire. 
Graphique 1. Proportion moyenne d’artistes Vivants de la mÊMe région QUe la STRUCTURE QUI LES RÉMUNÈRE, EN FONCTION DU CHIFFRE D'AFFAIRES DU DIFFUSEUR (MOYENNE SUR 3 ANS)

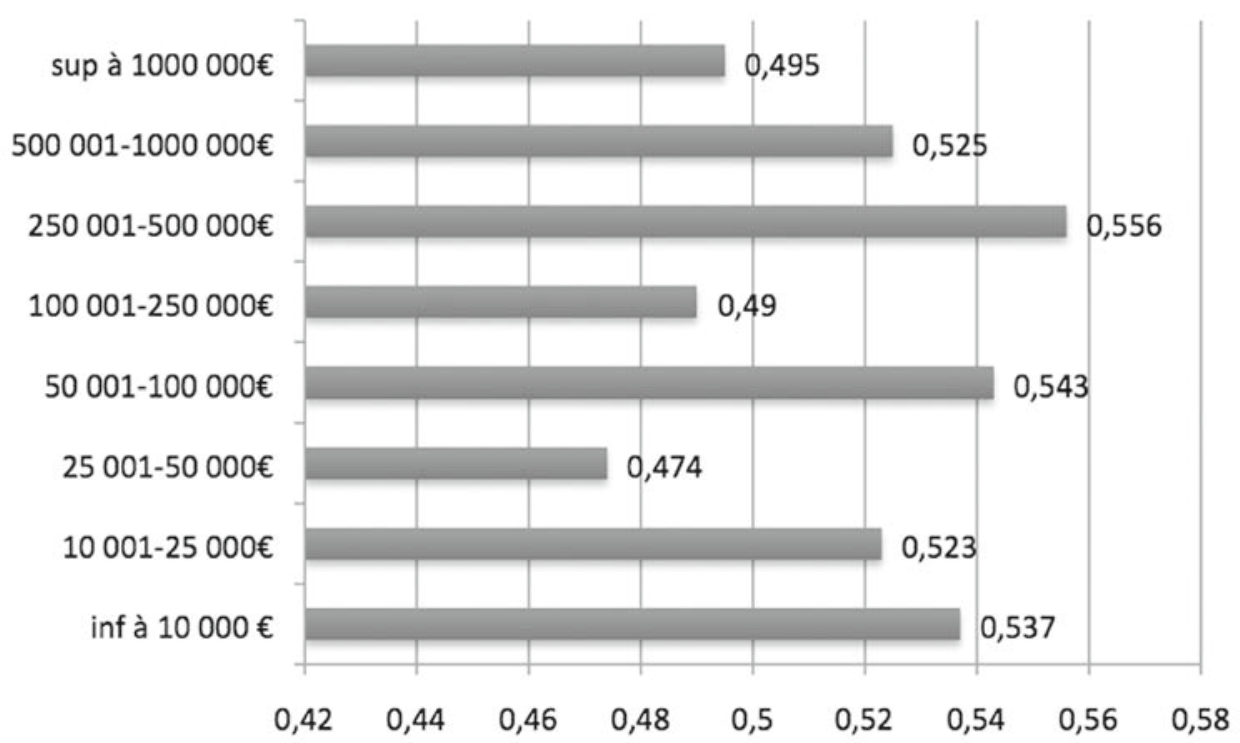

Ce premier volet d'analyse nous a permis de mettre à jour différents profils de galeries. Si toutes semblent chercher à se rapprocher géographiquement de bassins de clientèle denses, on voit apparaître quelques différences dans la prise de risque : sur Paris les galeries du quartier Matignon représentent ainsi des artistes plus anciens et ont un chiffre d'affaires plus élevé que leurs consœurs de l'Est Parisien. On note également un chiffre d'affaires plus faible des galeries provinciales qui cherchent à limiter le risque en jouant sur un portefeuille d'artistes plus élevé. Ces données ne nous disent rien cependant des potentielles collaborations des galeries entre elles. Une deuxième analyse plus ciblée sur différentes régions nous a permis de les mettre à jour via une analyse de réseau.

\subsection{Les résultats de l'enquête régionale : les enjeux de la proximité organisée}

D'après l'analyse des liens entre les structures de diffusion effectuée avec le logiciel Ucinet, il apparaît que dans les cinq agglomérations enquêtées, les acteurs de l'art actuel se polarisent autour de deux mondes, un monde institutionnel fortement structuré en réseau et un monde marchand, très éclaté et le plus souvent en marge des institutions et des politiques publiques.

Le schéma simplifié ci-après représente le mode d'organisation récurrent de l'art actuel dans les agglomérations étudiées. En gris clair, un réseau très dense se caractérise par de nombreuses coopérations entre acteurs. Des galeries le plus souvent associatives émanant 
d'anciens élèves des Beaux-Arts développent des liens de coopérations entre elles (nocturnes communes, organisation de salons, etc.). Elles tissent en outre des liens avec des acteurs institutionnels tels que l'École nationale supérieure des beaux-arts, le FRAC (Fonds régional d'art contemporain), les musées ou centres d'art contemporain ; s'adjoignent parfois à ce réseau quelques galeries privées de promotion, notamment à Lyon ${ }^{20}$. En gris foncé, des galeries commerciales représentant un art traditionnel sont très largement coupées du premier réseau, notamment du monde institutionnel et entretiennent peu de relations entre elles.

\section{SChÉma 1. Configuration SChÉmATIQUe DES MONDES DE L’ART ACTUEL EN RÉGION}
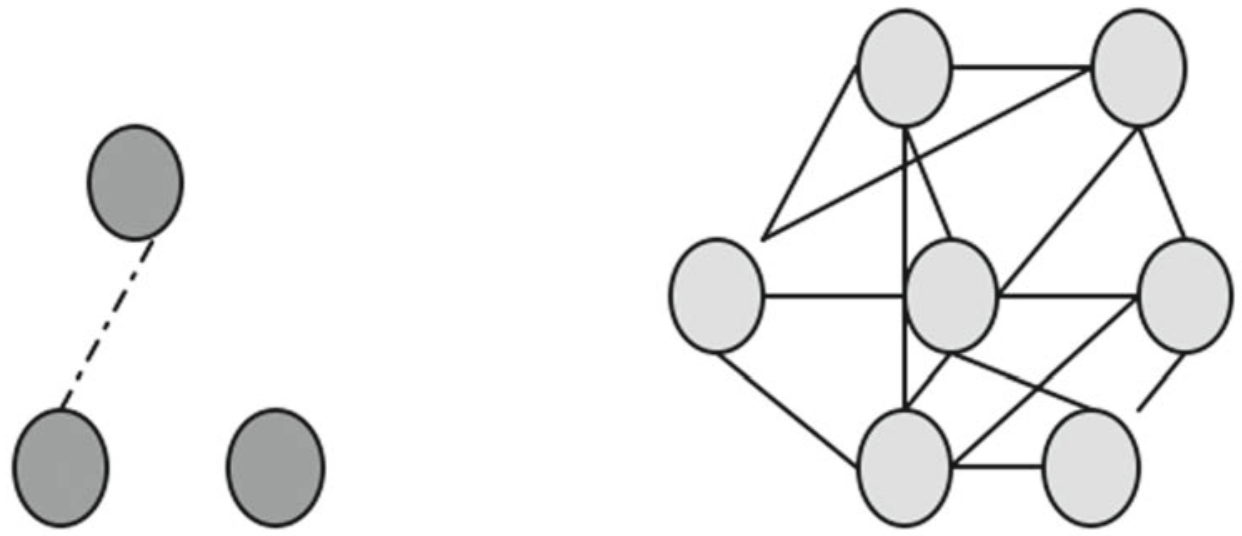

Gris clair : réseau institutionnel, art d'innovation

Gris foncé : galeries, art de tradition

N.B. : Les traits pleins indiquent que l'existence de liens a été constatée systématiquement dans chaque agglomération. L'existence de pointillés indique l'existence de liens pouvant apparaître entre acteurs, ceux-ci sont toutefois superficiels et non systématiques.

Au cours des entretiens, il est apparu que chaque famille fonctionnait de façon distincte, développant un discours qui peut aller jusqu'au rejet du travail effectué par les autres familles. Chacun possède ses événements propres, ses relais d’information. L’appartenance à une famille implique souvent l'impossibilité d'entrer dans les autres, les ponts existants entre les différents réseaux sont peu nombreux.

Les résultats tant de l'enquête quantitative que de l'enquête qualitative montrent que des forces distinctes sont à l'œuvre, certaines favorisant la concentration sur le territoire et la

20 Notons qu'à la marge de ce réseau, des galeries représentatives de ce que Nathalie Heinich (1998) qualifie d'art moderne tentent difficilement de garder contact avec le premier réseau, notamment par l'intermédiaire des associations des amis des musées et des services culturels des villes. Pour une présentation détaillée de cette organisation en réseau, voir De Vrièse, Martin, Moureau et SagotDuvauroux (2001b). 
collaboration entre firmes, d'autres agissant dans le sens contraire. La section suivante propose une discussion de ces résultats.

\section{DISCUSSION}

Nous commencerons par discuter les phénomènes de localisation des structures sur le territoire et les oppositions de profil qui semblent apparaître selon leur localisation géographique puis dans un second temps, nous mettrons en lumière comment le soutien d'un type d'art plutôt qu'un autre (proximité de valeur, convention de qualité artistique) favorise ou non la collaboration et la mise en réseau entre structures.

\subsection{Des externalités à la dépendance de sentier}

Concentration et différenciation, telles semblent être les caractéristiques de la localisation des galeries : concentration sur différents pôles d'attraction, mais dans le même temps, différenciation entre ces pôles.

Divers arguments peuvent être invoqués pour expliquer ces tendances.

En premier lieu, l'attraction des centres touristiques et des villes peut aisément s'expliquer par des arguments classiques, les firmes tendent à se regrouper naturellement auprès de lieux caractérisés par une demande locale importante ou richement dotés en ressources tangibles ou intangibles (Marshall, 1890 ; Porter, 1994).

Toutefois, d'autres forces jouent en sens inverse comme l'intensification de la concurrence et l'augmentation des loyers sur un site donné. Ces derniers éléments créent une dynamique de territoire en impulsant l'apparition de nouveaux foyers de concentration, caractérisés dans le domaine de l'art contemporain par la promotion de nouvelles esthétiques (stratégie de différentiation ${ }^{21}$ ) et l'aménagement de quartiers bon marché, accessibles aux jeunes galeries (Molotch et Treskon, 2009; Peterson, 1997 ; Benhamou, Moureau et Sagot-Duvauroux, 2001, 2002 ; Moureau et Sagot-Duvauroux, 2010). Le rôle centripète du prix des loyers qui conduit les galeries à s'éloigner des quartiers devenus trop chers se combine avec l'attrac-

21 Les travaux conduits sur les pratiques culturelles soulignent comment celles-ci sont concentrées au sein des grandes métropoles (Donnat, 2008). Le travail d'enquête a par ailleurs clairement mis en avant qu'il n'était pas rare que ces galeries tirent également parti de cet ancrage en développant un art lié à leur territoire. Pour certaines, c'est l'histoire de l'art qui permet de marquer ce positionnement, parce que certains mouvements historiques de l'art contemporain sont nés sur ce territoire (e.g. Support Surface ou la Figuration libre à Montpellier) ou parce que des écoles régionales s'y sont épanouies (école de Lyon, école de Nantes); pour d'autres, c'est le sujet traité qui rattache l'œuvre à la région (les falaises sur les côtes de Haute-Normandie, la cathédrale à Rouen, les marines et les vues portuaires à Nantes, le Pic Saint-Loup à Montpellier). Dans ce dernier cas, les œuvres sont acquises par les amateurs et collectionneurs du genre représenté, mais également les visiteurs de passage qui peuvent emporter un « souvenir » de la région. 
tion que joue la construction de nouveaux équipements culturels dans la capitale (Bastille, Beaubourg, Bibliothèque nationale). C'est ainsi qu'historiquement, une première vague de galeries s'est installée à la fin du $19^{\mathrm{e}}$ au sein du quartier Matignon puis, après la seconde guerre mondiale, le mouvement s'est déplacé Rive gauche dans le quartier de la rue de Seine. Le quartier Beaubourg a suivi à la fin des années 1960, puis Bastille dans les années 1980 ; à la fin des années 1990, cela a été le tour de la Bibliothèque François Mitterrand avec en particulier la rue Louise Weiss ${ }^{22}$. Ce résultat est cohérent avec les résultats de précédents travaux : tandis que dans une étude sur l'industrie audiovisuelle en Angleterre, Cook et Pandit (2004) mettent en exergue comment l'augmentation des loyers à Soho a poussé les firmes à se localiser ailleurs, Lazzeretti, Boix et Capone (2009) mettent en avant le rôle des dotations culturelles et historiques dans la concentration des firmes.

Ce déplacement géographique des galeries au cours de l'histoire contribue largement à expliquer les écarts que l'on observe selon les quartiers dans la façon dont les galeries constituent leurs portefeuilles d'artistes, illustrant un phénomène de dépendance de sentier. Les quartiers qui avaient été les premiers investis, comme Matignon, sont logiquement ceux où l'on retrouve les galeries ayant des portefeuilles mixtes d'artistes vivants/artistes décédés. À l'inverse, dans les quartiers Est, plus jeunes, les structures privilégient la vente exclusive d'œuvres d'artistes vivants (80,9\%).

\subsection{Des proximités de valeurs aux mondes de l'art}

Il peut paraître curieux qu’en dépit de proximités géographiques, certaines galeries ne se rencontrent pas, voire s'ignorent. Néanmoins, nombre d'auteurs soulignent que, pour qu'un rapprochement entre structures puisse être effectif, il importe que la proximité soit également organisée. «[...] Etre proche de quelqu'un, ce n'est pas seulement se trouver à côté de lui, ce peut être aussi avoir une forte connivence avec une personne géographiquement éloignée, qu'elle appartienne au même cercle d'amitié, familial, ou au même réseau d'entreprises ou professionnel »(Rallet et Torre, 2004, p. 25). S'agissant de l'art actuel, l'existence de plusieurs conventions de qualité a largement été approfondie (Heinich, 1998 ; Moureau et SagotDuvauroux, 1992, 2010 ; Martin, 2005) et s'inscrit dans la théorie des mondes de l'art de Becker (1982), selon laquelle coexistent plusieurs mondes de l'art, chacun fonctionnant selon ses propres conventions de production et de qualité, ses intermédiaires spécifiques ${ }^{23}$. D’un côté se trouvent des œuvres de facture traditionnelle tant par les matériaux employés que par les sujets traités, nécessitant souvent un savoir-faire artisanal de haut niveau, et pour

22 Notons que cette évolution n'est pas propre à Paris mais se retrouve à New York et Amsterdam (Crane, 1989; Velthuis, 2007 ; Moloch et Treskon, 2009).

23 Un monde de l'art est alors défini selon Becker par le "réseau de tous ceux dont les activités, coordonnées grâce à une connaissance commune des moyens conventionnels de travail, concourent à la production des cuvres qui font précisément la notoriété du monde de l'art » (Becker, 1982, p. 22). 
lesquelles la perception de la qualité ne requiert pas un travail d'intermédiation poussé24. De l'autre côté, se trouvent des œuvres dont la valeur est fondée sur l'originalité de la démarche artistique, il s'agit d'un art de recherche, de rupture par rapport à ce qui a été fait auparavant. Le discours importe tout autant que l'œuvre qui n'acquiert de sens qu'au sein de l'ensemble de la démarche de l'artiste.

L'existence de plusieurs registres d'évaluation de la qualité (convention classique versus convention d'originalité) a de fortes implications quant au rôle dévolu aux intermédiaires sur le marché et quant à la nature des coopérations et des intermédiations. Lorsque les acteurs ne partagent pas les mêmes conventions de qualité, il devient très problématique de développer des projets communs. La convention permet en effet de réduire l'incertitude, de sécuriser les anticipations et est source de proximité dans la mesure où le partage de la situation qu'elle permet favorise la coordination avec autrui. De ce fait, proximités spatiale et cognitive ne suffisent pas à produire de la coopération; celle-ci a également besoin d'une proximité de valeur (partage d'une même convention de qualité) comme le montre l'enquête. Des galeries proches géographiquement ne développent aucune coopération entre elles, car elles ne partagent pas de proximité de valeur ; cette dernière apparaît alors comme une condition nécessaire à la coopération.

Revenons au schéma présenté dans la précédente section (cf. encadré 1). Les galeries qui développent peu de réseaux de coopération sont celles qui relèvent de la convention classique. Pour ce type de galeries, la qualité est intrinsèque aux œuvres, elle renvoie à des critères purement techniques (qualité de la facture par exemple) et au registre émotionnel. De fait, le rôle de la galerie consiste à opérer une première sélection et à combler l'écart géographique existant entre l'artiste et le collectionneur. Le galeriste, qui se trouve en concurrence monopolistique avec ses confrères, réduit au maximum les collaborations, celles-ci se traduisant par exemple dans le cadre de la ville de Montpellier à l'édition d'un dépliant commun disponible à l'office du tourisme.

En revanche, les galeries qui promeuvent un art de recherche, innovant, sont insérées dans un réseau complexe. L'évaluation de la qualité de ces œuvres est moins simple que dans le cas précédent, elle n'est plus intégrée à l'œuvre, mais relève d'un dispositif de légitimation qui requiert l'intervention de nombreux acteurs appartenant pour partie au monde institutionnel et pour partie au monde marchand (Moulin, 1992 ; Moureau et Sagot-Duvauroux, 2010). Dans ce cas, la fonction d'intermédiation qualitative des galeristes est cruciale. Ceuxci conduisent un travail spécifique afin d'insérer le travail des artistes qu'elles représentent dans un réseau de légitimation institutionnel. Des coopérations sont développées avec d'autres galeries ainsi qu'avec des institutions telles que les musées (édition de catalogue en partenariat, exposition conjointe, achat), les FRAC (acquisition ou exposition), les organes du ministère de la Culture au travers de subventions (aide à la première exposition, à l'édition, soutien pour une participation aux foires, etc.) et les centres d'art labellisés. Les entretiens conduits nous ont permis de constater qu'en région, les galeries qui soutiennent cet art de recherche ont essentiellement un statut associatif (pouvant ainsi bénéficier de subventions et vivre en dépit de peu de ventes). Elles offrent généralement aux nouveaux talents une

24 Ce qui renvoie à l'art classique défini par Heinich (1998) ou au marché des chromos de Raymonde Moulin (1967). 
première exposition, mais n'ont pas vocation à les conserver dans leur portefeuille d'artistes sur le long terme. Elles jouent en quelque sorte un rôle de tremplin en offrant aux artistes les moyens d'étoffer leur Curriculum vitae en début de carrière. Néanmoins, il importe qu'après ces premières expositions, ils trouvent d'autres relais plus pérennes, ouverts sur d'autres territoires et marchés.

Si le partage de valeur apparaît comme une condition nécessaire au développement de collaborations, notons que dans le cas de "l'art de recherche ", il constitue également une des conditions vitales de l'existence des galeries, celles-ci n'étant pas à même par leur seule action d'offrir au public des signaux suffisamment crédibles quant à la qualité des œuvres qu'elles proposent ${ }^{25}$.

\section{CONCLUSION}

Cet article avait pour objectif d'expliquer la forte concentration géographique des galeries d'art et dans quelles conditions cette concentration pouvait déboucher sur des coopérations. Nous avions posé l'hypothèse que l'existence de coopération nécessitait que la proximité spatiale des galeries soit accompagnée d'une proximité organisée et en particulier d'une proximité de valeur. La forte concentration géographique des galeries d'art s'explique par l'importance des effets d'agglomération dont bénéficient ces très petites entreprises : externalités d'information, économie de transports pour les fournisseurs et les clients, valorisation des aménités patrimoniales. Cependant, l’augmentation du coût des loyers et la nécessité, pour les nouvelles galeries de se différencier des plus anciennes, s'est traduit à Paris par une dynamique d'implantation dans de nouveaux quartiers, caractérisés par des loyers plus faibles et bien souvent par l'implantation d'un nouvel équipement patrimonial susceptible d'attirer des visiteurs. Nous avons également mis en évidence sur les différents territoires explorés, une segmentation du marché en deux mondes très étanches. Nous avons montré alors, conformément à notre hypothèse de départ, l'importance des proximités de valeurs pour le développement ou non de coopérations. Il apparaît ainsi que pour les galeries tournées vers un art de recherche, l'organisation en réseau est nécessaire pour insérer le travail des artistes qu'elles défendent dans un maillage tant marchand qu'institutionnel (Fonds régional d'art contemporain [FRAC], École nationale supérieure des beaux-arts, etc.). Denses, les liens de coopération entre les structures du réseau institutionnel se concrétisent par des coéditions de catalogues, des échanges ou l'organisation d'expositions communes, l'accueil d'artistes en résidence. La coopération l'emporte sur la compétition (coopétition). Les galeries qui appartiennent au monde de l'art traditionnel utilisent pour leur part les seules formes de coopérations qui leur sont stratégiquement utiles, à savoir l'édition de dépliants communs distribués par des offices de tourisme ou en des signalisations sur les sites des collectivités publiques. La compétition l'emporte sur la coopération.

25 Notons que dans ce cas, la constitution du Curriculum vitae joue un rôle essentiel dans la carrière des artistes, tout autant que l'œuvre produite, car il constitue un certificat de qualité, précieux, attestant des multiples étapes franchies et de la reconnaissance de leur travail par les instances de légitimation (diplôme des Beaux-Arts, présence à des foires, galeries qui les ont représentés, expositions dans des lieux labellisés, etc.). 
À l'issue de cette analyse, il apparaît que loin d'être des structures « sans calcul » et des entreprises qui adhèrent à la philosophie de l'art for art sake, les galeries d'art contemporain savent tirer parti du territoire en fonction de leur intérêt stratégique.

\section{RÉFÉRENCES}

Becker, H. (1982). Art Worlds. Oakland, University of California Press.

Bellet, M., Colletis, G. et Lung, Y. (dir.) (1993). Économie de proximités. Revue d'économie régionale et urbaine, 3(numéro spécial), 357-361.

Bellet, M., Kirat, T. et Largeron, C. (1998). Approches multiformes de la proximité. Paris, Hermès.

Benhamou, F., Moureau, N. et Sagot-Duvauroux, D. (2001). Les galeries d'art contemporain en France. Paris, La Documentation française.

Benhamou, F., Moureau, N. et Sagot-Duvauroux, D. (2002). Opening the black box of the white cube : a survey of french contemporary art galleries at the turn of the millenium. Poetics Journal of Empirical Research on Culture, Media and the Arts, 30(4), 263-280.

Boschma Ron, A. (2005). Proximity and innovation : a critical assessment. Regional Studies, 39(1), 61-74.

Bouba-Olga, O. et Grossetti, M. (2008). Socio-économie de proximité. Revue d'économie régionale et urbaine, 2008(3), 311-328.

Caves, R. (2000). Creative industries, contracts between art and commerce. Cambridge, Harvard University Press.

Chesnel, S., Molho, J., Morteau, H. et Raimbeau, F., et Sagot-Duvauroux, D. (2013). Les clusters ou districts industriels du domaine culturel et médiatique : revue du savoir économique et questionnement. Paris, France, Ministère de la Culture et de la Communication.

Cook, G.A.S. et PAndit, N.R. (2004). Clustering in the british broadcasting and financial services industries : a comparative analysis of three regions. Problems and Perspectives in Management, 2004(3), 72-88.

CRAne, D. (1989). The transformation of the avant garde. Chicago, University of Chicago Press.

De Vrièse, M., Martin, B., Moureau, N. et Sagot-Duvauroux, D. (2011a). Portrait économique des diffuseurs d'art actuel inscrits à la Maison des artistes (Culture chiffres). Paris, Ministère de la Culture (DEPS).

De Vrièse, M., Martin, B., Moureau, N. et Sagot-Duvauroux, D. (2011b). Diffusion et valorisation de l'art actuel en région. Une étude des agglomérations du Havre, de Lyon, de Montpellier, de Nantes et de Rouen (Culture études). Paris, Ministère de la Culture (DEPS).

Donnat, O. (2008). Les pratiques culturelles des français à l’heure du numérique. Paris, La Découverte.

Dubuisson-Quellier, S. (2003). Contacts et relations au marché chez les très petites entreprises. Réseaux, 21(121), 19-42.

FILLIS, I. (2002). Barriers to internationalization : an investigation of the craft microentreprise. European Journal of Marketing, 36(7-8), 912-927.

Gilly, J.P. et Torre, A. (2000). Dynamiques de proximité. Paris, L'Harmattan. 
Gordon, I.R. et McCann, P. (2000). Industrial clusters : complexes, agglomeration and/or social networks? Urban Studies, 37(3), 513-532.

Greffe, X. et Simonnet, V. (2008). La survie des entreprises culturelles : le rôle du regroupement géographique. Recherches économiques de Louvain, 74(3), 327-357.

GUNDOLF, K. (2006). L'interaction des stratégies individuelles et collectives des très petites entreprises: étude comparée des sites touristiques de montagne (thèse de doctorat en sciences de gestion). Université de Montpellier I, France.

Gundolf, K. (2008). Cohésion territoriale et proximité des acteurs : le cas des TPE de tourisme. Dans A. Jaouen. et O. Torrès, Les très petites entreprises (p. 27-55). Cachan, Éditions Lavoisier.

Gundolf, K. et Jaouen, A. (2005). Patterns and coordination of collective action in small and very small business : the case of a tourist village in the Pyrenees. International Journal of Entrepreneurship and Small Business, 2(3), 392-403.

Gundolf, K., JAouen, A. et Loup, S. (2006). Institutions locales et TPE dans le cas du tourisme. Revue française de gestion, 32(167), 141-157.

Heinich, N. (1998). Le triple jeu de l'art contemporain. Paris, Les Éditions de Minuit.

JACKson, J. et Murphy, P. (2002). Tourism destinations as clusters : analytical experiences from the new world. Tourism and Hospitality Research, 4(1), 36-52.

JaOUen, A. et ToRrès, O. (2008). Les très petites entreprises. Cachan, Éditions Lavoisier.

Kosianski, J.-M. (2011). Territoire culture et politiques de développement économique local : une approche par les métiers d'art. Revue d'économie régionale et urbaine, 2011(1), 81-111.

Kusin, D. (2005). The modern and contemporary art market. Helvoirt, Pays-Bas, The European Fine Art Foundation.

Lazzeretti, L., Boix, R. et CApone, F. (2009, juin). Why do creative industries cluster? An analysis of the determinants of clustering of creative industries. Papier présenté à la DRUID Summer Conference. Copenhague, Danemark.

Loup, S. (2003). Les petites entreprises des métiers d’art. Revue française de gestion, 114(3), 195-209.

Loup, S., Jourdain, A., Kosianski, J.-M. et Rakotovahino, M.-A. (2010). Les apports de la pluridisciplinarité à la connaissance d'un propriétaire dirigeant de très petite entreprise : le professionnel des métiers d'art. $10^{\circ}$ Congrès international francophone en entreprenariat et PME. Bordeaux, France.

Mallard, A. (2007). La pluralité des rapports au marché dans les très petites entreprises : une approche typologique. Économie et statistique, 407(1), 51-71.

Marshall, A. (1890). Principles of economics. Londres, Macmillan.

Martin, B. (2005). L'évaluation de la qualité de l'art contemporain: le cas de l'insertion des jeunes artistes plasticiens (thèse de doctorat en sciences économiques). Université Paris $\mathrm{X}$, France.

Molotch, H. et Treskon, M. (2009). Changing art : Soho, Chelsea and the dynamic geography of galleries in New York city. International Journal of Urban and Regional Research, 33(2), 517-541.

Moulin, R. (1967). Le marché de la peinture en France. Paris, Minuit.

Moulin, R. (1992). L'artiste, l'institution et le marché. Paris, Flammarion.

Moureau, N. et Rivaud-Danset, D. (2010). La nature versus l'expert ou le paradoxe du marché de l'art contemporain. Dans A. Hatchuel, F. Favereau, F. Aggeri et E. Coblence (dir.), L'activité marchande sans le marché (p. 285-295). Paris, Presses de l'École des mines. 
Moureau, N., Sagot-Duvauroux, D. (1992, octobre). Les conventions de qualité sur le marché de l'art, d'un académisme à l'autre? Esprit, (10) 43-54.

Moureau, N. et Sagot-Duvauroux, D. (2010). Le marché de l'art contemporain. Paris, La Découverte.

Pecqueur, B. et Zimmermann, J-B. (2004). Les fondements d'une économie de proximité. Dans B. Pecqueur et J-B. Zimmermann (dir.), Économie des proximités (p. 13-41). Paris, Hermès.

Peterson, K. (1997). The distribution and dynamics of uncertainty in art galleries : a case study of new dealerships in the parisian art market, 1985-1990. Poetics, 25(4), 241-263.

Pilati, T. et Tremblay, D.-G. (2007). Cités créatives et districts culturels : une analyse des thèses en présence. Géographie économie et société, 9(4), 381-401.

Porter, M. (1994). The role of location in competition. Journal of the Economics of Business, 1(1), 35-39.

Rallet, A. et Torre, A. (1995). Économie industrielle et économie spatiale. Paris, Economica.

Rallet, A. et Torre, A. (2004). Proximité et localisation. Économie rurale, 280(1), 25-41.

Santagata, W. (2006). Cultural districts and their role in developed and developing countries. Dans V.A. Ginsburgh et D. Throsby (dir.), Handbook on the economics of art and culture (p. 1101-1119). Oxford, Elsevier.

Scotт, A.J. (2010). Creative cities : the role of culture. Revue d'économie politique, 120(1), 181-204.

Scott, A.J. et LeRiche, F. (2005). Les ressorts géographiques de l'économie culturelle : du local au mondial. L'Espace géographique, 34(3), 207-222.

Talbot, D. et Kirat, T. (2005). Proximités et institutions : nouveaux éclairages. Économie et institutions, (6-7), 75-100.

Torre, A. (2006). Clusters et systèmes locaux d'innovation : retour critique sur les hypothèses naturalistes de transmission des connaissances à l'aide des catégories de l'économie de la proximité. Région et développement, (24), 15-44.

Torre, A. et Rallet, A. (2005). Proximity and localization. Regional Studies, 39(1), 47-60.

Velthuis, O. (2007). Talking prices : symbolic meanings of prices on the market for contemporary art. Princeton, New Jersey, Princeton University Press.

Zukin, S. (2011). The life cycle of New York's creative districts : reflections on the unanticipated consequences of unplanned cultural zones. City, Culture and Society, 2(3), 131-140. 